\title{
Rama Muscular Hipertrófica de la Arteria Recurrente Radial (Cuerda de Henry) en Relación con el Ramo Profundo del Nervio Radial. Reporte de Caso
}

\author{
Hypertrophic Muscular Branch of Radial Recurrent Artery (Leash of Henry), \\ in Relation to the Deep Branch of Radial Nerve. Case Report
}

Carreño-Vásquez, Andrés A.

CARREÑO-VÁSQUEZ, A. A. Rama muscular hipertrófica de la arteria recurrente radial (Cuerda de Henry) en relación con el ramo profundo del nervio radial, Reporte de caso. Int. J. Morphol., 38(4):853-856, 2020.

RESUMEN: La arteria recurrente radial nace en el extremo proximal de la arteria radial y desde ahí asciende oblicuamente para anastomosarse con la arteria colateral radial, entregando en ese trayecto una serie de ramas para los músculos cercanos. Dicha arteria junto con sus ramas fueron descritas (por su importancia en abordajes quirúrgicos) por Arnold K. Henry como "the radial leash". Actualmente en clínica se utiliza el nombre "leash of Henry" para referirse a una o más ramas musculares de la arteria recurrente radial, sobretodo cuando cuando se encuentran en relación con el ramo profundo del nervio radial, pudiendo llegar a causar compresiones de dicho nervio en algunos casos. Se realizó una descripción de caso de una leash of Henry atípica, encontrada en una muestra cadavérica del laboratorio de anatomía de la Universidad Católica del Maule, de sexo masculino y nacionalidad chilena. La arteria encontrada corresponde a la rama de mayor calibre de la arteria recurrente radial, que se dirige directamente al músculo extensor de los dedos, dibujando un trayecto horizontal y cruzando por anterior al ramo profundo del nervio radial. Esta hallazgo difere a lo descrito por Henry y otros autores más recientes, y por lo tanto aporta información potencialmente útil a la hora de realizar procedimientos quirúrgicos que requieran un abordaje posterior o lateral de la cabeza del radio, como también descompresiones del nervio radial en esta zona.

PALABRAS CLAVE: Arteria recurrente radial; Cuerda de Henry; Nervio radial; Codo.

\section{INTRODUCCIÓN}

La arteria recurrente radial (ARR) nace en el extremo proximal de la arteria radial a nivel de la fosa del cubital, sitio desde el cual asciende oblicuamente hasta que se anastomosa con la rama anterior de la arteria colateral radial, participando en las anastomosis periarticulares del codo, en ese trayecto la ARR proporciona ramas musculares a los músculos vecinos (Rouvière et al., 2005; Moore et al., 2013).

Por otro lado, el músculo extensor de los dedos corresponde a un músculo epicondíleo lateral ubicado en la capa superficial del compartimento posterior de antebrazo, medial al músculo extensor radial corto del carpo. Este músculo se encuentra irrigado normalmente por ramas de la arteria interósea posterior (Latarjet et al., 2019) y de acuerdo con Testut \& Latarjet (1973), a esta última se podría añadir la arteria interósea recurrente.

En esta región es importante describir al ramo profundo del nervio radial (RPNR), que en búsqueda del com- partimento posterior del antebrazo, se dirige hacia el margen superior aponeurótico del fascículo superficial del músculo supinador, sitio donde ingresa a un intersticio celular delgado ubicado entre los fascículos superficial y profundo de dicho músculo, y del cual emerge como nervio interóseo posterior (Rouvière et al.), este margen aponeurótico es conocido clínicamente como la "arcada de Frohse" (AF) y en este sitio el RPNR se puede encontrar en una estrecha relación con estructuras vasculares provenientes de la ARR (Hanna, 2015), correspondiendo también a la zona donde dicho nervio es anatómicamente más vulnerable, ya sea por estructuras musculares, bandas fibrosas o vasos sanguíneos de la zona (Kim et al., 2017).

Esta relación del RPNR con estructuras vasculares, proximal a la AF, fueron descritas por Arnold K. Henry en su libro "Extensile Exposure", quien describe a la ARR bajo el nombre "the radial leash" haciendo referencia no a un único vaso arterial, sino a un grupo de vasos de los cuales el 
más proximal es recurrente y el resto corresponden a pequeñas pero importantes ramas musculares que se distribuyen en forma de abanico (fan-like) hacia los músculos braquiorradial y los extensores radiales largo y corto del carpo, anclándose en ellos y distribuyéndose en distintos planos musculares, de esta manera actúan como un medio de fijación para dichos músculos y necesarios de tener a estos vasos en cuenta a la hora de realizar abordajes quirúrgicos del radio (Henry 1970/1957).

Esta descripción de importancia clínica-quirúrgica de la distribución de la ARR, llevó a que los clínicos usaran el nombre "leash of Henry" (LH) o en español "cinturón de Henry" para referirse a una o más ramas arteriales provenientes de la ARR (o la ARR en si misma), sobretodo cuando estos vasos se encuentran en relación con el RPNR proximal a la AF.

\section{MATERIAL Y MÉTODO}

La muestra corresponde a un cadáver perteneciente a la Unidad de anatomía de la Facultad de Medicina, Universidad Católica del Maule, Talca, de sexo masculino, chileno, fallecido en el 2010 a la edad de 55 años, causa de muerte desconocida.

Este hallazgo fue obtenido a partir de una disección realizada en una muestra intervenida previamente con fines de docencia, la cual se encontraba parcialmente disecada. De esta manera se delimitaron cada uno de los músculo superficiales del antebrazo y se realizó una incisión con separación entre los músculos extensores radiales del carpo y el músculo extensor de los dedos, una vez realizado dicho abordaje se observó al músculo supinador y la AF, visualizando inmediatamente la presencia de un vaso arterial de tamaño significativo, que cruzaba transversal y anteriormente al RPNR, una vez identificado este vaso se procedió a ampliar el campo desinsertando a los músculos superficiales.

Una vez obtenido una amplia y clara visión del campo intervenido, se procedió a medir la longitud y diámetro de la arteria con un cáliper digital, y relaciones con estructuras óseas, musculares y nerviosas vecinas, tomándose la fotografía correspondiente y finalmente se realizó un dibujo esquemático de la misma.

Cabe destacar que las mediciones, fotografía y dibujo fueron realizados con el antebrazo en pronación, dado que clínicamente esta es una de las posiciones utilizada cuando se requiere un abordaje quirúrgico posterior o lateral del radio, donde se ubica el antebrazo del paciente en esta posi- ción para exponer el compartimento extensor de antebrazo y además ubicar, aislar y rechazar al RPNR manteniéndolo dentro del campo visual durante la cirugía y protegiéndolo de cualquier lesión (Testut \& Jacob, 1972; Hoppenfeld et al., 2005).

\section{RESULTADOS}

La arteria encontrada corresponde a la rama de mayor tamaño de la ARR, que aparece recorriendo un trayecto transversal de medial a lateral, a la altura del cuello del radio, en búsqueda del músculo extensor de los dedos. Dicho recorrido se presentó a $8,4 \mathrm{~mm}$ por debajo de la cabeza del radio, 15,0 mm desde la interlínea articular radiohumeral y a 36,0 mm de distancia del epicóndilo lateral del húmero (Fig. 1).

La ARR entregó previamente dos ramas arteriales de menor tamaño para los músculos extensor radial largo del carpo y extensor radial corto del carpo, junto a una pequeña rama para el músculo braquiorradial, a su vez la rama muscular del extensor de los dedos entregó una pequeña rama en su origen y otra aun más pequeña un poco antes de introducirse en el dicho músculo. Dejando de lado estas dos pequeñas ramas, esta arteria dibuja un trayecto horizontal uniforme e ininterrumpido en su recorrido.

El diámetro de la ARR fue de 3,3 mm en su origen y de 2,1 mm inmediatamente distal al nacimiento de la rama muscular encontrada, esta última presentó un diámetro de 2,6 mm en su origen y 2,1 mm en su extremo distal, antes de introducirse dentro del músculo extensor de los dedos.

El largo de la ARR desde su origen hasta que proporciona la rama muscular del extensor de los dedos fue de 19 $\mathrm{mm}$ y el largo de esta última desde su origen hasta ingresar dentro de dicho músculo fue de $36 \mathrm{~mm}$.

\section{DISCUSIÓN}

La información que se puede encontrar en general sobre la ARR en los textos utilizados normalmente para el estudio de la anatomía (Moore et al.) o incluso en textos clásicos como el de Testut \& Latarjet o Hamilton et al. (1965) no aportan mayores detalles sobre esta arteria (fuera de su anastomosis con la colateral radial), ni es posible encontrar descritas las relaciones de dicha arteria con las estructuras nerviosas cercanas (RPNR) o una descripción en detalle de sus ramas, pero mediante el estudio de los dibujos y esque- 

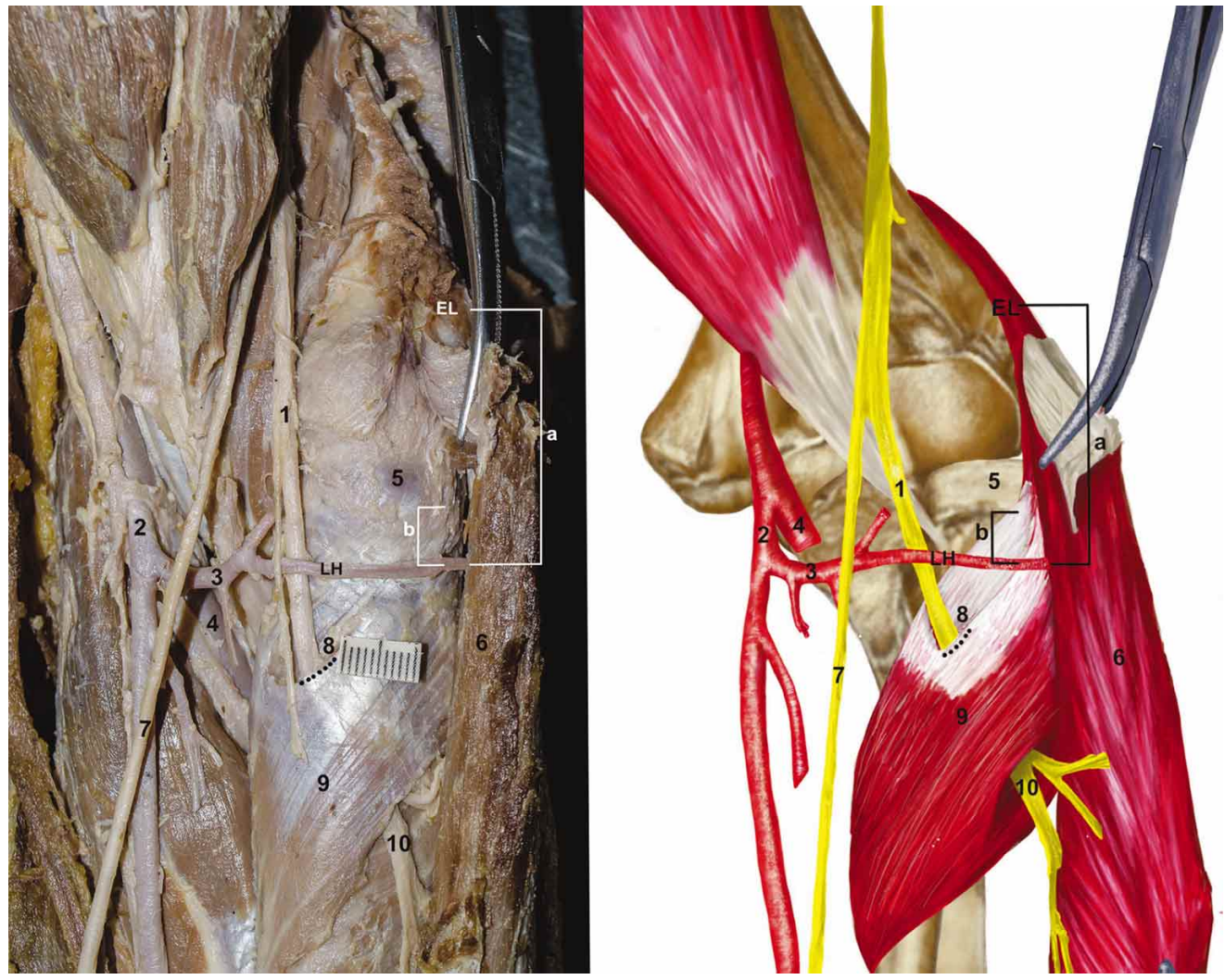

Fig. 1. Fotografía y esquema de disección en la fosa cubital con antebrazo en pronación (Se han removido los mm. braquiorradial, extensor radial largo y corto del carpo. 1. Ramo profundo del nervio radial; 2 . A. radial; 3. A. recurrente radial; 4. A. ulnar; 5. cabeza del radio; 6. M. extensor común de los dedos; 7. Ramo superficial del nervio radial; 8. Arcada de Frohse; 9. M. supinador; 10. n. Interóseo posterior. LH. Leash of Henry; EL. Epicóndilo lateral. La distancia entre EL y LH está representada en " $A$ " y fue de $36,0 \mathrm{~mm}$, mientras que la distancia comprendida entre 5 y LH está representada en "B" y fue de 8,4 $\mathrm{mm}$.

mas presentes en dichos textos, siempre se ve que la ARR o alguna de sus ramas pasan posterior al ramo profundo del nervio radial o medial a este y con un trayecto oblicuamente ascendente en todos los casos, lo que contrasta con este hallazgo que aporta información distinta al respecto de la arteria y su relación con el RPNR, pasando transversal y anteriormente a dicho nervio.

Respecto a la irrigación del músculo extensor de los dedos, se describe normalmente a la arteria interósea posterior como la responsable de entregar dicho aporte sanguíneo (Latarjet et al., 2019) y solo Standring (2016) reconoce a la arteria recurrente radial como la encargada de la irrigación del tercio proximal de dicho músculo (y el resto por la interósea posterior), lo cual sería concordante con lo encontrado en esta muestra.

En cuanto a lo descrito por Henry (1970), este caso se presenta distinto, dado que, más que una serie de pequeñas ramas en forma de abanico, predomina el tamaño de una de dichas ramas por sobre el resto, irrigando además a un músculo que no aparece dentro del texto de Henry como irrigado por la ARR.

Con respecto a las relaciones que se pueden establecer entre LH y el RPNR, Tubbs (2006) encontró estruc- turas vasculares (LH) cruzando al RPNR en 33 de 34 de las muestras analizadas, con una distancia de entrecruzamiento con dicho nervio, medida desde el epicóndilo lateral, de $35 \mathrm{~mm}$ a $60 \mathrm{~mm}$, por lo que nuestro caso $(36 \mathrm{~mm}) \mathrm{se}$ encontraría dentro del rango de menor distancia de entrecruzamiento con el RPNR tomando como referencia dicho punto óseo.

Por otro parte, la importancia clínica de este hallazgo se debe a que a nivel de la $\mathrm{AF}$ se encuentra el sitio donde con mayor frecuencia se producen compresiones del nervio radial (dentro de la región antebraquial) ya sea como consecuencia de la propia AF (con mayor frecuencia) o debido a la presencia de bandas fibrosas, quistes, vasos radiales recurrentes, entre otros (Martinoli et al., 2004; Kim et al.). Sobre esta última causa, algunos autores detallan específicamente como causa vascular de compresión del RPNR, al cruce del nervio por una leash of Henry en condición de "Hipertrófica" (Xiao \& Cartwright, 2019).

$\mathrm{Al}$ respecto no está claro en la literatura cuando considerar a una LH como hipertrófica, por ejemplo, Husarik et al. (2009) estableció como criterio al hallazgo de seis o más vasos alrededor del RPNR proximal a la AF, mientras que Loizides et al. (2011) describe dos casos de compresión del ramo profundo del nervio radial a causa de una 
única LH hipertrófica (detectado a través imágenes de ultra sonido de alta resolución), pero sin detallar el criterio utilizado para dicha consideración. En otro estudio, Breglia et al. (2015) encontró 4 de 17 casos de compresión del RPNR a causa de vasos radiales recurrentes sin detallar si existía hipertrofia o no.

Ante la ausencia de un criterio único y claro, he clasificado como hipertrófica a esta rama muscular del extensor de los dedos, tomando en cuenta que es la rama de mayor calibre de la ARR y que presenta un ancho mayor (en su origen) que la continuación del tronco principal del cual proviene (2,6 $\mathrm{mm}$ y $2,1 \mathrm{~mm}$ respectivamente).

Este hallazgo aporta información potencialmente útil a la hora de realizar procedimientos quirúrgicos que incluyan un abordaje posterior o lateral de la cabeza del radio como también en descompresiones del RPNR a nivel de la AF donde esta rama muscular (o ramas) denominadas como "Leash of Henry" pudiese ser la causa de la compresión o presentar un riesgo quirúrgico. Por lo tanto, dado que se presenta un caso atípico según lo que se encuentra ya descrito en la literatura y estudios previos, es importante tener en cuenta la variabilidad de este vaso en cuanto a su tamaño y distribución.

CARREÑO-VÁSQUEZ, A. A. Hypertrophic muscular branch of radial recurrent artery (leash of Henry), in relation to the deep branch of radial nerve, Case report. Int. J. Morphol., 38(4):853$856,2020$.

SUMMARY: The radial recurrent artery originates at the proximal end of the radial artery and from there ascends obliquely to anastomosing with the radial collateral artery. It gives off several branches for nearby muscles on its path. This artery along with its branches were described (due to its importance in surgical approaches) by Arnold K. Henry as "the radial leash". Currently, in clinical terms, the name "Leash of Henry" is used to refer to one or more muscular branches of the radial recurrent artery, especially when they are in relation to the deep branch of the radial nerve, and may cause compression of the nerve in some cases. A case description of an atypical Leash of Henry was found, found in a Chilean, male cadaveric sample of the anatomy laboratory, Universidad Católica del Maule. The artery corresponds to the branch of greater caliber of the recurrent radial artery, which goes directly to the extensor digitorum muscle. It draws a horizontal path and crosses the deep branch of the radial nerve anteriorly. This finding differs from what was described by Henry and other more recent authors. Therefore, this is potentially useful information when performing surgical procedures that require a posterior or lateral approach to the radius head, as well as radial nerve decompressions in this area.

KEY WORDS: Radial recurrent artery; Leash of Henry; Radial nerve; Elbow.

\section{REFERENCIAS BIBLIOGRÁFICAS}

Breglia, G. A.; Fracalossi, M. \& Destaillats, A. Síndrome del tunel radial. Hallazgos y tratamientos en 17 pacientes. Rev. Asoc. Argent. Ortop. Traumatol., 80(3):158-63, 2015.

Hamilton, R.; Hamilton, G. \& Fyfe, F. Anatomía Humana. Ciudad de México, Interamericana, 1965.

Hanna, A. S. Anatomy and Exposures of Spinal Nerves. Cham, Springer, 2015.

Henry, A. K. Extensile exposure. $2^{\text {nd }}$ ed. London, Churchill Livingstone, 1970.

Hoppenfeld, S.; Hutton, R.; Thomas, H. A. \& DeBoer, P. Abordajes en Cirugía Ortopédica. Madrid, Marbán, 2005.

Husarik, D. B.; Saupe, N.; Pfirrmann, C. W.; Jost, B.; Hodler, J. \& Zanetti, M. Elbow nerves: MR findings in 60 asymptomatic subjects--normal anatomy, variants, and pitfalls. Radiology, 252(1):148-56, 2009.

Kim, Y.; Ha, D. H. \& Lee, S. M. Ultrasonographic findings of posterior interosseous nerve syndrome. Ultrasonography, 36(4):363-9, 2017.

Latarjet, M.; Ruiz Liard, A. \& Pró, E. Anatomía Humana. $5^{\text {a }}$ ed. Buenos Aires, Médica Panamericana, 2019.

Loizides, A.; Peer, S.; Ostermann, S.; Henninger, B.; Stampfer-Kountchev, M. \& Gruber, H. Unusual functional compression of the deep branch of the radial nerve by a vascular branch (leash of Henry): ultrasonographic appearance. Rofo, 183(2):163-6, 2011.

Martinoli, C.; Bianchi, S.; Pugliese, F.; Bacigalupo, L.; Gauglio, C.; Valle, M. \& Derchi, L. E. Sonography of entrapment neuropathies in the upper limb (wrist excluded). J. Clin. Ultrasound, 32(9):438-50, 2004.

Moore, K.; Dailey, A. \& Agur, A. Anatomía con Orientación Clínica. $7^{\text {a }}$ ed. Barcelona, Wolters Kluwer Health, 2013.

Rouvière, H.; Delmas, V. \& Delmas, A. Anatomía Humana Descriptiva, Topográfica y Funcional. 11ª ed. Barcelona, Elsevier Masson, 2005.

Standring, S. Gray's Anatomy. The Anatomical Basis of Clinical Practice. $41^{\mathrm{a}}$ ed. Philadelphia, Elsevier, 2016.

Testut, L. \& Jacob, O. Tratado de Anatomía Topográfica Con Aplicaciones Médico-Quirúrgicas. Barcelona, Salvat Editores, 1972.

Testut, L. \& Latarjet, M. Tratado de Anatomía Humana. $9^{\mathrm{a}}$ ed. Barcelona, Salvat Editores, 1973.

Tubbs, R. S.; Salter, E. G.; Wellons, J. C. 3rd; Blount, J. P. \& Oakes, W. J. Superficial surgical landmarks for identifying the posterior interosseous nerve. J. Neurosurg., 104(5):796-9, 2006.

Xiao, T. G. \& Cartwright, M. S. Ultrasound in the evaluation of radial neuropathies at the elbow. Front. Neurol., 10:216, 2019.

Dirección para correspondencia:

Andrés Carreño Vásquez

Departamento de Ciencias Preclínicas

Facultad de Medicina

Universidad Católica del Maule

Av. San Miguel, 3605

Talca

CHILE

Email: acarreno@ucm.cl

Recibida : 04-10-2019

Aceptada: 06-03-2020 